\title{
八肽胆囊收缩素对羟甲芬太尼镇痛的拮抗 作用可以被氯化锂所翻转*
}

韩念霜 张丽娟 韩济生

(北京医科大学生理教研室, 北京 100083)

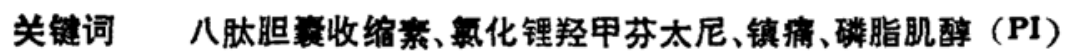

许多报道已经表明, 阿片类物质的作用 (如镇痛, 摄食等) 可以被八肽胆衰收缩素（CCK8) 所对抗, 但其机制不明. CCK-8 的抗阿片作用可以发生在受体水平 ${ }^{[1]}$, 也有可能发生在受 体后第二信使水平. 在外周组织, 特别是在胰腺的外分泌细胞, CCK-8 能刺激肌醇磷脂转化 产生肌醇三磷酸 ( $\mathrm{Ins}_{3}$ ) 和甘油二酯 (DAG); 这两种第二信使可以使细胞内的 $\mathrm{Ca}^{2+}$ 储存释 放并激活蛋白激酶 $\mathrm{C}(\mathrm{PKC})^{[2]}$. 虽然外周与中枢的 $\mathrm{CCK}-8$ 在受体机制和生理功能方面有很 多不同之处,我们仍然考虑到,在外周由 CCK-8 所引起的受体后信息传导系统是否也在中枢 神经系统内发生.

已知锂盐能干扰肌醇磷脂的转化 ${ }^{[3]}$, 本文中我们观察氯化锂是否能影响中枢 CCK-8 的 抗阿片活性. 本实验室以往的研究表明, CCK-8 的抗阿片效应选择性地作用于 mu 和 $\mathrm{kappa}$ 受体, 而不作用于 delta 受体 ${ }^{[1]}$, 所以在我们的研究中不用吗啡而用羟甲芬太尼, 后者是一种 具有高度选择性的 $\mathrm{mu}$ 受体激动剂.

\section{1 材料与方法}

\section{1 动物实验方法}

脊髓蛛网膜下腔 (ith) 埋置瘘管 ${ }^{[4]}$ ：200-250g 雄性 Wistar 大鼠在水合氯醛麻醉下 $(0.4 \mathrm{~g} / \mathrm{kg}$, i.p. ) , 于枕骨下缘依次切开皮肤、肌肉、宮枕膜和硬脊膜. 经切口向脊髓蛛网膜下 腔插人外径 $0.61 \mathrm{~mm}$ 的 PE-10 聚乙烯管, 插人长度为 $7.5 \mathrm{~cm}$, 达到脊髓腰膨大处, 皮肤外留 $6 \mathrm{~cm}$, 术后 $24 \mathrm{~h}$ 开始实验. 经插管注人药液 $10 \mu 1$, 继以生理盐水 $10 \mu \mathrm{l}$ 冲洗管内残留溶液.

测痛 ${ }^{[5]}$ : 将大鼠装人特制塑料圆筒内, 尾和四肢露在筒外. 待动物安静 $30 \mathrm{~min}$ 后, 以辐 射热甩尾法测痛. 用 $8.75 \mathrm{~mm}$ 放映灯泡发出的直径约 $4 \mathrm{~mm}$ 的光束 照射距尾尖部 3.5 至 $4.5 \mathrm{~cm}$ 处的尾部皮肤; 测定甩尾反应潜伏期 (TFL), 间隔 $5 \mathrm{~min}$ 后再测, 取三次测定的平均 值为基础痛阈 (一般为 4-6s), 给药处理后再测痛阈, 与基础 TFL 相比求得痛阈变化百分 数. 为避免灼伤, 以痛阈升高 $+150 \%$ 为上限, 超过 $+150 \%$ 时停止照射.

数据处理和统计学检验: 文中结果以均值士标准误 $(X \pm S E)$ 表示, 组间差异棌用方差 分析,进一步用 Duncan's Test 多重检验. 以 $p<0.05$ 作为显著性差异的标准。

1992-04-21 收稿, 1992-10-22 收修改稿.

*国家自然科学基金资助项目. 


\section{2 药品和试剂}

羟甲芬太尼 (Ohmefentanyl, OMF) 为中国科学院上海药物研究所产品.八肽胆囊收缩 素 (CCK-8: Asp-Tyr-Met-Gly-Trp-Met-Asp-Phe）为美国 Squibb 药厂馈赠. 水合氯 醛、氮化锂为北京化工厂产品.

\section{2 实 验 结 果}

\section{$2.1 \mathrm{LiCl}$ 对大鼠基础痛阈的影响}

取 6 只大鼠,测定基础痛阈后, 每隔 $10 \mathrm{~min}$ ith 注射一次 $\mathrm{LiCl}$, 其剂量分别为 $1.25,2.5$,

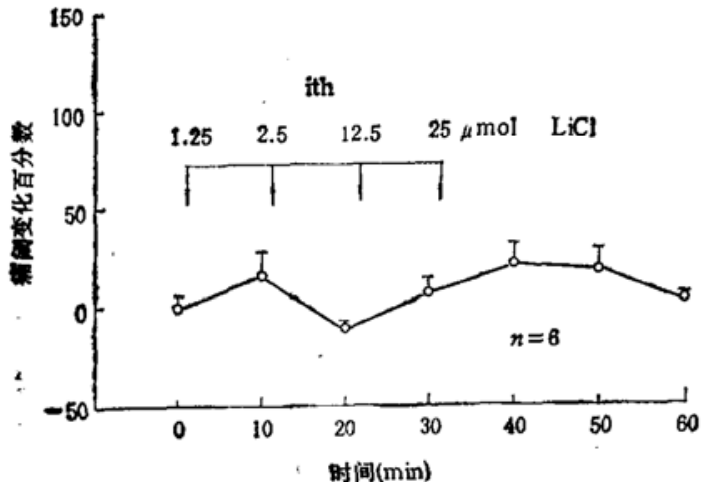

图 1 脊顝蛛网膜下腔累加注射 $\mathrm{LiCl}$ 对基础痛阈的影响

*为每组动物数. j $j$ 差分析 (ANOVA) 表明, $\mathrm{LiCl}$ 对 痛阈的影响并无统计学显著性 $12.5,25 \mu \mathrm{mol}$. 从第一次注射起, $60 \mathrm{~min}$ 内 TFL 无明显改变(见图 1).

\section{$2.2 \mathrm{LiCl}$ 对䍩甲芬太尼镇痛的影响}

将 14 只大鼠随机分为两组,测定基础痛 间后 ith 注射 $\mathrm{mu}$ 阿片型受体激动剂 OMF $20 \mathrm{ng}, 10 \mathrm{~min}$ 即引起痛阈明显升高, 此后每 隔 $10 \mathrm{~min}$ 累加注射 $\mathrm{NS}$ 或 $\mathrm{LiCl} 0.3,0.6$, $1.25,2.5 \mu \mathrm{mol}$ (容积均为 $10 \mu \mathrm{l}$ ). $70 \mathrm{~min}$ 内, $\mathrm{LiCl}$ 组与 $\mathrm{NS}$ 对照组相比, TFL 变化 百分数无显著差异 (图 2a).

另取大鼠 54 只, 分成 6 组. 在 ith 注射 $20 \mathrm{ng}$ OMF 后 $10 \mathrm{~min}$, 再次注射 NS（即 $\mathrm{OMF}+\mathrm{NS}$ 对照组), 或不同剂量的 $\mathrm{LiCl}$

$(1.25,2.5,5,10,20 \mu \mathrm{mol})$, 测定首次注射后 20-50min 内痛闻变化的百分数, 取其平均值, 结果见图 2b. OMF + NS 对照组痛阈平均升高 $70+18.3 \%$, 注射 $\mathrm{LiCl}$ 的各组痛阈升高值 在 $55 \pm 5 \%$ 和 $89 \pm 22 \%$ 之间, 与 OMF + NS 组相比, 差异无显著意义.
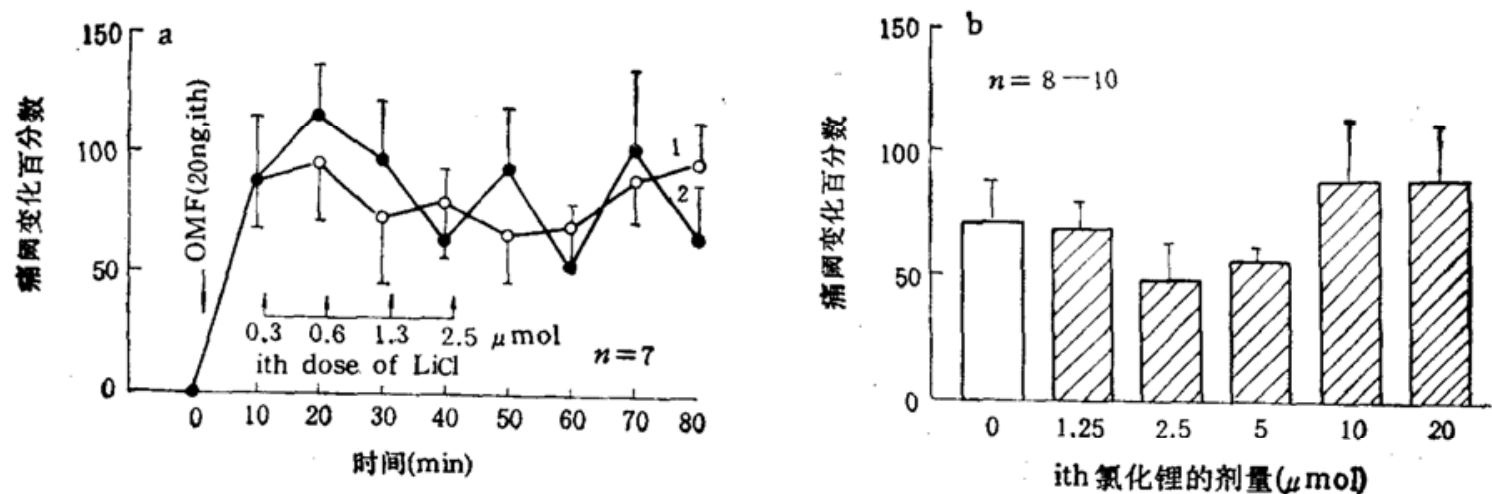

图 2

8. 脊融蛛网膜下腔 (ith) 累加注射 $\mathrm{LiCl}$ 对 OMF (20ng, ith) 镇窝效应并无显著影响. $n$ 为每组 动物数. ANVOA, $p>0.05 、 1-\mathrm{OMF}(20 \mathrm{ng}), 2-\mathrm{OMF}+\mathrm{LiCl}$;

b. ith 注射不同剂量的 $\mathrm{LiCl}$ 对 OMF (20ng, ith) 镇痛效应的影响. 在 ith 注射 $20 \mathrm{ng}$ OMP $10 \mathrm{~min}$ 后注射 $\mathrm{LiCl}$. 图中纵坐标为注射 $\mathrm{LiCl}$ 后 $20 \mathrm{~min}$ 内四次测痛所得痛阈变化百分数的平均值, 竖线示标准误,各组之间差异无显著性 (ANOVA， $p>0.05$ )

这些结果表明, 给动物急性注射 $\mathrm{LiCl}$ 对基础痛阈没有影响, 对 $\mathrm{mu}$ 型阿片受体激动剂 
OMF 引起的镇痛作用也无显著影响.

\subsection{CCK-8 对抗 OMF 的镇痛作用}

ith 注射 $20 \mathrm{ng}$ OMF 后 $10 \mathrm{~min}$, 再次 ith 注射 NS 或不同剂量的 CCK-8 $(1,2,4,8$, $16 \mathrm{ng}$ ). 测定 $20--50 \mathrm{~min}$ 内 $\mathrm{TFL}$ 变化百分数的平均值. 结果见表 1. 不同剂量的 CCK -8 能够不同程度地翻转 OMF 的镇痛作用, 将各翻转百分数作直线回归得相关系数 $r=0.84$. 对该回归直线进行拟合优度检验， $p<0.05$. 由回归方程计算出 翻转 OMF 镇痛 $50 \%$ 所需 CCK -8 的剂量为 $8 \mathrm{ng}$.

表 1 CCK-8 拮抗 OMF 的镇痛作用“

\begin{tabular}{c|c|c|c|c|c|cc}
\hline & \multicolumn{5}{|c}{ CCK-8 (ng, ith) } \\
\cline { 2 - 6 } & NS & 1 & 2 & 4 & 8 & 16 \\
\hline TFL (\% chang) & $75 \pm 21$ & $64 \pm 5$ & $55 \pm 20$ & $21 \pm 12$ & $31 \pm 11$ & $26 \pm 2$ \\
$\%$ Reversal & 0 & 15 & 26 & 72 & 59 & 65 \\
N & 10 & 9 & 8 & 7 & 11 & 9 \\
\hline
\end{tabular}

a) 表中所列数据为注射 OMF (20 ng, ith) 后 20-50 min 内, 甩尾潜伏期变化百分率的平均值士标准误, OMF 和 CCK-8 分别在 $0 \mathrm{~min}$ 与 $10 \mathrm{~min}$ 时注射.

\subsection{CCK-8 对抗 OMF 统裙的作用可被 $\mathrm{LiCl}$ 转}

四组大鼠 ith 注射 $20 \mathrm{ng}$ OMF 后 $10 \mathrm{~min}$, 分别 ith 注射 $\mathrm{NS} 10 \mu \mathrm{l}, \mathrm{CCK}-88 \mathrm{ng}, \mathrm{LiCl}$ $1.25 \mu \mathrm{mol}$ 或 $\mathrm{CCK}-8$ 加 $\mathrm{LiCl} 1.25 \mu \mathrm{mol}$, 测定 $70 \mathrm{~min}$ 内痛阈变化结果见图 3. OMF 能引 起 TFL 明显升高,至少保持 $60 \mathrm{~min}$. CCK-8 能明显对抗 OMF 的镇痛作用, 且 CCK-8 的 作用至少持续 $50 \mathrm{~min}$. 与 OMF + NS 对照组对比, 差异非常显著 $(p<0.01) ; \mathrm{LiCl}$ 本身 对 OMF 的镇痛作用无明显影响 (注射 $\mathrm{LiCl} 30 \mathrm{~min}$ 后痛间似略有降低, 但无统计学意义 $(p>0.05)$. 但当 $\mathrm{CCK}-8$ 和 $\mathrm{LiCl}$ 同时注射时, $\mathrm{OMF}$ 的镇痛作用又重新表现出来, 换言 之, CCK-8 对抗 OMF 镇痛的效应完全被 $\mathrm{LiCl}$ 所翻转(与单纯 CCK-8 组相比, $p<0.01$; 但与 OMF 对照组相比, $p>0.05$ ).

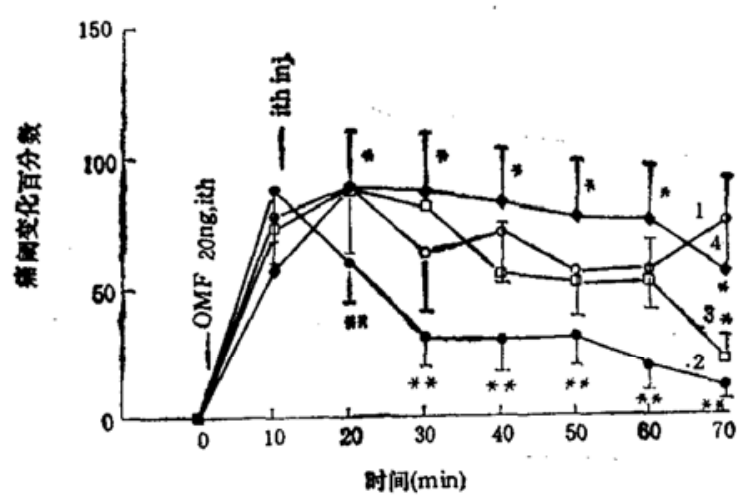

图 3 CCK-8 拮抗 OMF 镇痛的效应被芧化锂所翻转 与 $\mathrm{OMF}+\mathrm{NS}$ 组相比较, ${ }^{*} p<0.01$, 与 $\mathrm{OMF}+\mathrm{CCK}$ 组相比较, ** $p<0.01 .1-$ NS $10 \mu 1(10), 2-\mathrm{CCK}-8 \mathrm{8ng}(11), 3-\mathrm{LiCl} 1.25 \mu \mathrm{mol}(8), 4-\mathrm{CCK}+$ LiC1 (7), 括号内为每组动物数, 坚线示标准误

为探索 $\mathrm{LiCl}$ 翻转 CCK-8 作用的量效关系, 在 ith 注射 OMF $20 \mu \mathrm{g}$ 后 $10 \mathrm{~min}$, 再次 
ith 注射不同剂量的 $0.3-20 \mu \mathrm{mol} \mathrm{LiCl}$ 和 $8 \mathrm{ngCCK}-8$ (总体积 $10 \mu \mathrm{l}$ ), 取注射 $\mathrm{OMF}$ 后 20-50 min TFL 变化百分数的平均值,结果见图 4. 图 4 中第一条空心柱表示单纯 OMF 的 镇痛效果. 第二条斜线柱表示 CCK 对 OMF 镇痛的对抗作用. 在此基础上, 加用低剂量 $(0.3 \mu \mathrm{mol}) \mathrm{LiCl}$ 不能对抗 CCK -8 的作用;在略微增大剂量后 $(0.6 \mu \mathrm{mol}$ 以上), CCK -8 的 作用即被取消. 在 $0.6-10 \mu \mathrm{mol}$ 范围内, $\mathrm{LiCl}$ 的作用基本保持在目标水平. 剂量加大到 $20 \mathrm{~mol}$ 时,不仅翻转了 $\mathrm{CCK}-8$ 的抗阿片作用,还加强 OMF 的镇痛效应.

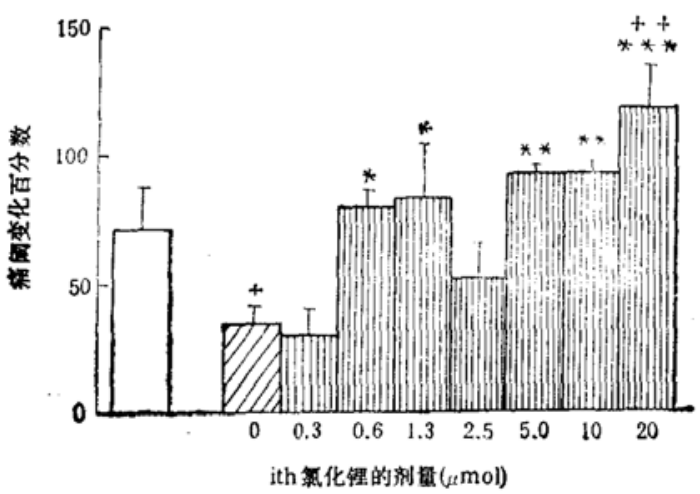

图 4 不同剂量的氯化锂对 $\mathrm{CCK}-8$ 抗阿片效应的影响 ith 注射 OMF $20 \mathrm{ng}$ 后 $10 \mathrm{~min}$, ith 注射 NS, 或 CCK-8 $8 \mathrm{ng}+\mathrm{NS}$ 或 CCK$88 \mathrm{ng}+\mathrm{LiCl}$. 纵坐标为注射 OMF 后 20-50 min 期间四次测痛痛酎变化百分数 的平均值, $\square$ OMF (20ng,ith) + NS, VID OMF + CCK $(8 \mathrm{ng}, \mathrm{ith})+\mathrm{NS}$, 而W $\mathrm{OMF}+\mathrm{CCK}+\mathrm{LiCl}, n=8-10, n$ 为每组动物数. 竖线示标准误, 经 ANOVA 继以 Duncan 检验, 与 OMF + NS 对照组相比较, + 表示 $p<0.05,++$ 表示 $p<0.01$, 与 OMF + CCK 组相比较, ${ }^{*} p<0.01, * * p<0.05, * * * p<0.001$

\section{3 讨 论}

尽管在临床上锂盐已经成功而广泛地应用于治疗躁狂-抑郁型精神病有 30 多年的历史， 但对于锂盐在中枢神经系统内的作用机制,至今尚不清楚.早在 1971 年, Allison 和 Stewart 就报道了锂盐能抑制肌醇磷酸酶的活性 ${ }^{[6]}$. 以后越来越多的证据表明锂盐能够通过选择性抑 制肌醇磷酸酶, 从而耗竭脑内自由肌醇的含量来干扰磷脂肌醇的转化 ${ }^{[3]}$.

许多研究表明,在外周组织中,细胞内肌醇的来源主要有三种峜径:（1）从细胞间质摄取 来自血浆中的肌醇; (2) 重新利用肌醇磷酸水解释放的肌醇; (3) 由葡萄糖经 D-肌醇-3-磷酸 从头合成. 在 CNS, 由于肌醇难以跨过血脑屏障, 因此神经细胞只有由有限的胞外肌醇供

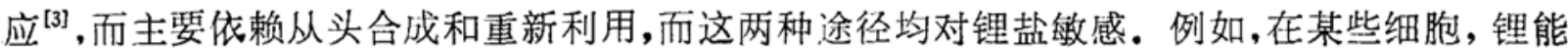
抑制肌醇合成过程中的关键酶-D-肌醇-3-磷酸酶的活性 ${ }^{[3]}$. 而在肌醇磷脂䛇环中, 锂还能抑 制去磷酸化酶(InsP 磷酸酶和多磷酸肌醇 1-磷酸酶)，从而减少肌醇的供应 ${ }^{[7]}$. 因此, “肌醇耗 竭假说”解释锂的作用是基于这样一个观点：锂能够通过降低脂质前体的供应而间接地抑制 信号传递系统,而脂质前体是产生第二信使物质 $\operatorname{lns}_{3}$ 和 DAG 所必须的 ${ }^{[3]}$.

与上述提到的假说一致,有人报道, 锂能明显抑制 $M$-胆碱能和 $\mathrm{a}$-肾上腺素能激动剂引起 的 $\mathrm{InsP}_{3}$ 的生成 ${ }^{[8]}$. 用 $\mathrm{M}$ 激动剂 Carbachol ( CCh) 刺激大脑皮层切片,发现锂增加 InsP, 但 抑制 Ins $(1,4,5) \mathrm{P}_{3}$ 和 Ins $(1,3,4,5) \mathrm{P}_{4}$ 的积累 ${ }^{[9]}$,这些研究表明在皮层组织,至少在体外实 验中,锂通过阻断了肌醇的供应, 可以影响到 PI 的代谢; 最近我室张丽娟等在酶法分离的新生 
鼠全脑细胞中观察到 CCK-8(10 nmol) 影响 InsP 和 InsP $\mathrm{P}_{3}$ 含量的效应与 CCh 极为相似 (待发表),表明 $\mathrm{CCh}$ 与 $\mathrm{CCK}-8$ 的作用机制很可能是类似的.

我们的实验表明, 脊髓蛛网膜下腔注射氯化锂对基础痛阈和 mu 阿片受体激动剂 OMP 所引起的镇痛均无影响, 但能十分明确地阻断 CCK-8 对于 OMF 镇痛的对抗作用. 鉴于锂 能干扰激动剂刺激的肌醇磷脂代谢, 因此本实验提示我们在 CCK-8 的抗阿片机制中, 肌醇磷 脂信号系统的活动可能起着关键性作用.

必须指出, 锂的作用是比较复杂的. 它对 PI 系统的影响随所观察的组织以及实验条件下 PI 系统受激动(磷脂酶被激活)的程度而不同. 被研究的组织中, 肌酻的供应途径和对激素的 感的 $\mathrm{PIP}_{2}$ 储存量的高低等因素影响实验结果. 因此本文提出的论点尚需用进一步的实验加 以验证.

致谢 实验中所用 CCK-8 是由美国 Squibb 公司馈赠,特此致谢.

\section{参考文献}

[1] Wang, X. J., Wang, X. H., Han, J. S., Brain Res., 1990, 523: 5-10.

[2] Berridge. M. J., The Scient. Am., 1985, 253: 124-134.

[ 3 ] Berridge, M. J., Dowmes, C. P., Hanley, M. R. Biochem J., 1982, 206: 587-595.

[4] Yaksh, T. L., Rudy, T. A., Science, 1976, 192: 1357-1358.

[5] 任民峰、韩济生,生理学报, 1978,30: 204-208.

[6] Allison, J. H., Stewart, M. A., Nature, 1971, 233: 267--268.

[7] Halleher, L. M., Sherman, W, R., J. Biol. Chem., 1980, 225: 10896-10901..

[ 8 ] Batty, I. R., Nahorski, S. R., J. Neurochem, 1985, 45: 1514-1521.

[9] Kennedy, E. D., Challiss, R. A. J., Nahurski, S. R., J. Neurochem., 1989, 53: 1652-1653. 